\section{Timely intervention helps protect the sexual health of adolescents}

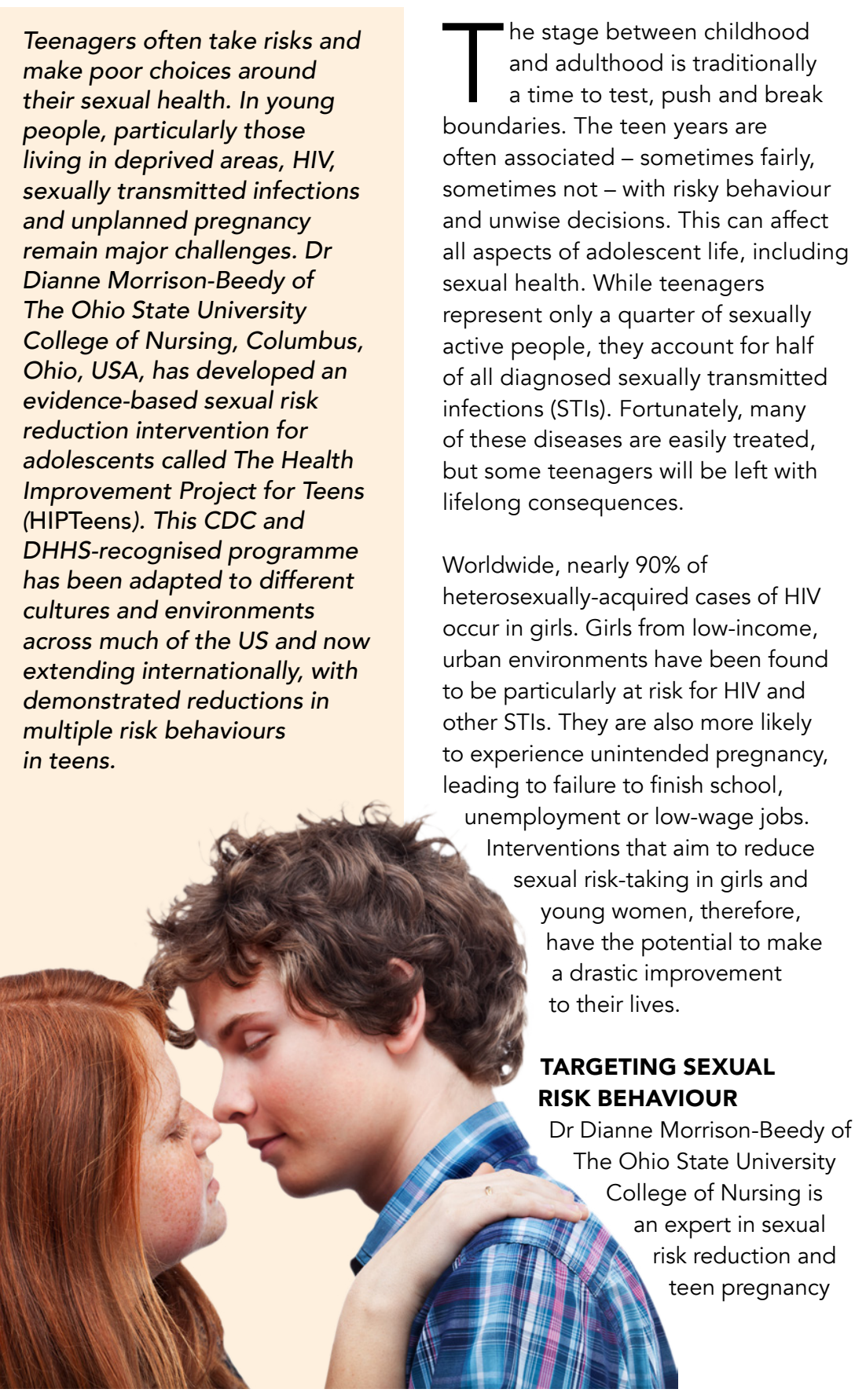

prevention. Several years ago, DrMorrison-Beedy led a large, investigate how effective a text insestigate how effective a sexua be in reducing sexul risk benavio in adelescent girls. With very litte research addressing this population The Health Improvement Project for Teens (HIPTeens) addressed the issue of both starting and maintaining needed risk-reduction practices. The trial was particularly focused on sexually-active girls from low-income, urban areas. In total, 738 girls aged between 15-19 participated in the study. They were predominantly $(69 \%)$ African American and impoverished with an average age of 16.5 years.

The research examined whether a gender-specific, theoretically-driven sexual risk-reduction (SRR) intervention " cossions, was effective in "booster" exual behaviour in the participants. The intervention took the form of four weekly two-hour sessions, held in small groups facilitated by trained femaleleaders from diverse backgrounds. The participants were provided with information on HIV/STIs and other sexual health topics, delivered in the form of games and other interactive activities. "Booster" sessions, lasting 90 minutes, were given 3 and 6 months after the initial intervention. A control group received the same number of sessions, but they focused on ther health issues, such as nutrition

Dr Morrison-Beedy hypothesised that the participants in HIPTeens would
HIPTeens is effective, in part, because
it targets the motivations behind risky
behaviour.

have less unprotected sex and fewer partners than those in the control is just what happened: the girls who received the HIPTeens intervention reduced several sexual risk behaviours over the following year. They were more likely to be abstinent and reduced their total sexual episodes compared to the control group. There were also fewer pregnancies in the HIPTeens group during the year. While STI rates were unchanged this was likely due to the entire group.

This trial demonstrated that brief, tailored sexual risk reduction boosterioss, combined with behaviour in a grou of sexually disenfranchised adolescent girls.

\section{HIPTEENS: TACKLING SEXUAL}

RISK IN TEENAGERS

Using the valuab from the original study, Dr MorrisonBeedy went on to refine and develop strategies to move this effective and critically needed intervention from research into communities. On average, it takes seventeen years to move findings from research into practice, and Dr Morrison-Beedy was committed and successful in accelerating the translational process in less the five years Nochly the project is recognised by the Centers for Diserse Control and Prevention (CDC) and the US Department of Health and Human Services (DHHS), and has been proven to reduce multiple risky behaviours in teens, which lead to STI and HIV transmission, as well as preventing teenage pregnancy, and staff across organisations can be quickly trained to provide HIPTeens.

HIPTeens is effective, in part, because it targets the motivations behind risky behaviour. In 2017, Dr Morrison-Beedy and her team returned to the data collected in the original study to delve deeper into the motivations behind the sexual choices of the participants.

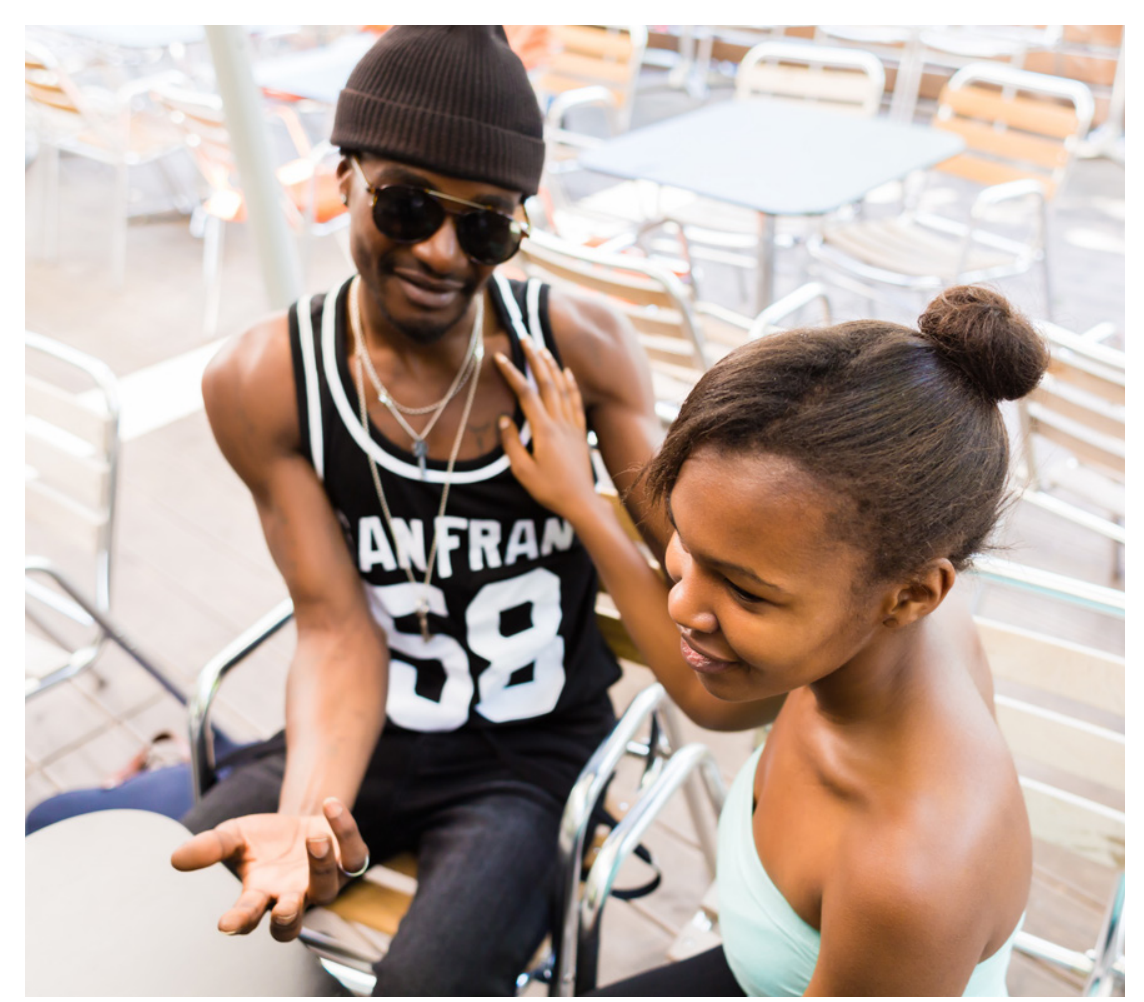

The girls and young women who received

The Health Improvement Project for

Teens (HIPTeens) intervention reduced their risky sexual behaviour.

who expressed motivations for sex as a way to cope with negative emotions, appease their partner, or boost their confidence or perceived attractiveness, were also likely to show symptoms of helth issues are an increasin

for adolescent girls combined with the findings from this study on motivations driving sexual decision-making, suggests that interventions, such as HPTeens, that tackle these negative in sexur risk reducto ineeded

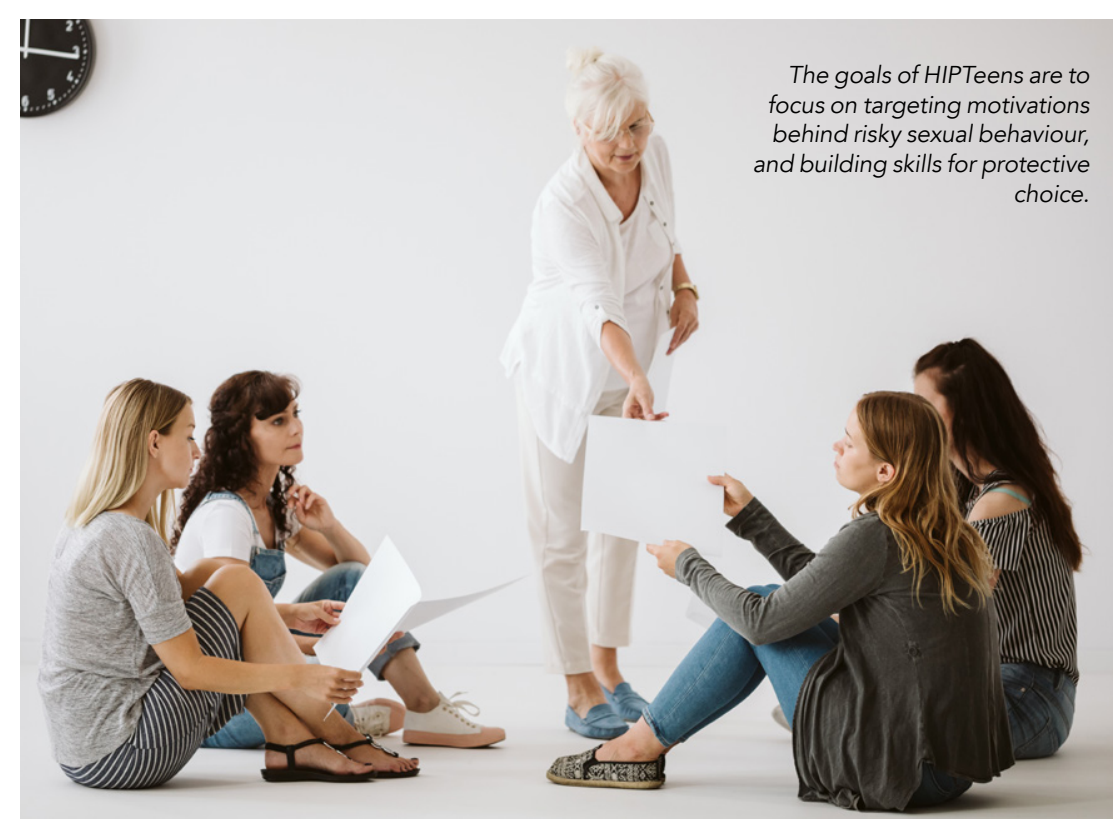




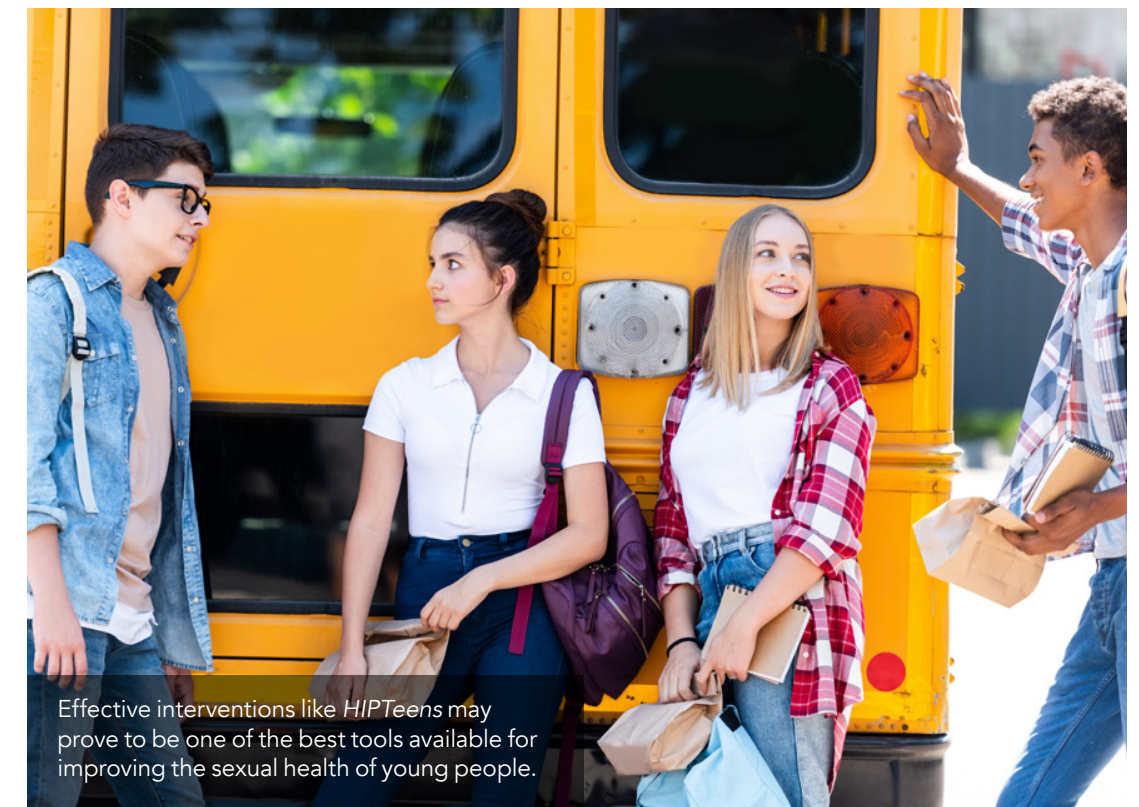

Knowing that large numbers of teens events, the research team have woven whether an intervention like HIPTeen would be useful in that environment. Despite a number of new measures those who will provide HIPTeens and materials in the intervention have been tailored using universal design for learning (UDL) to improve access for teens with intellectual and learning
disabilities. HIPTeens is a completely to protect people over the last decade, STI and HIV infections and unplanned pregnancy remain a problem among many Scottish youth. As in other parts of the world, including the US,

HIPTeens is effective because it targets both the motivations behind risky choices while providing needed skills

for communication, negotiation, and building resilience.

manualised, medically accurate intervention with fidelity assessments to mme is provided as developed.

\section{TAKING HIPTEENS INTERNATIONAL} HIPTeens was developed to be easily implemented and used by sttings that serve adolescent girls in need of a brief, tailored intervention that works. It has been adapted to the specific challenges and cultural background of girls and young wom different parts of the world and across different settings. In 2017 Dr Morrison-Beedy led a study in Edinburgh, Scotland to assess the

teenagers in economically deprived Understanding the knowledge, motivations and skills for protective behaviours in Scottish teenagers is the first step towards adapting an intervention like HIPTeens to the specific needs of the population.

The Edinburgh study included both male and female teenagers between the ages of 16 and 19. In total, eighteen young people took part in a series of questionnaires and focus group activities, during which the team asked a range of open-ended questions on sexual health issues. For example, the participants were given enough information on HIV ind STs, and where they got that greed that having the facts was not the same as knowing how to actually practice safer behaviours. They also revealed the challenges that drugs and alcohol contribute to unhealthy sexual behaviour choices and that substance use and sex are not connected in educational curriculum on health topics in school.

One important point uncovered by the Edinburgh project was that the scottish teenagers perceived themselves to be far less able to talk openly about sex, compared with the gmerican counterparts. Most felt that gaining skils in communication and safe opportunities to practice these skills in a programme, are important; but missing, experiences as they try to navigate their sexual health choices. Many also reported that many of their sexual risk behaviours took place in public settings and that often led to difficulty in discussing or assuring consent of their partners.

The specific circumstances, attitudes and experiences of the participants in the Edinburgh study highlighted the variety of ways in which HIPTeens might need to be adapted to remain relevant in different environments. Nevertheless, the goals of HIPTeens - to focus on targeting motivations behind risky sexual behaviour, and building skills for options - are universally relevant The programme can be adapted to groups of different sizes, ages, genders, and sexual orientation, and is rooted in years of scientific evidence.

Despite the medical advances of the twentieth and twenty-first centuries HIV and other STIs, and unintended pregnancy, remain very real challenges in the lives of many teenagers. Effective interventions like HIPTeens may prove to be one of the best tools health of young people.

For more information about the HIPTeens project, visit http://www. hip4change.com/

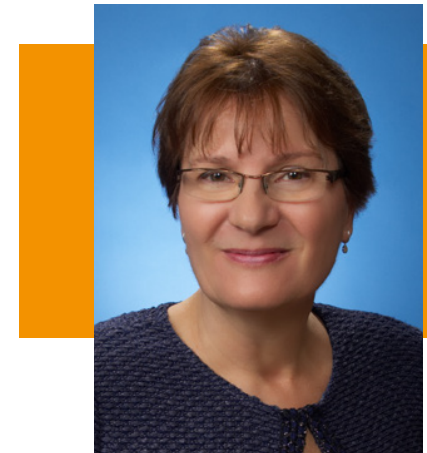

\section{Behind the Research}

\section{Dr Dianne Morrison-Beedy}

: morrisonbeedy@gmail.com T: +1-813-230-3335 W: https://www.hip4change.com

Research Objectives

Dr Morrison-Beedy developed HIPTeens through extensive, rigorous research; it is recognised as an exemplary HIV/STI/ Pregnancy evidence-based intervention.

\section{Detail}

Dianne Morrison-Beedy, PhD, RN, FNAP, FAANP, FAAN Chief Talent \& Global Strategy Officer

Cof Nursing

College of Nursing

760 Kinnear Road, \#223, Columbus, $\mathrm{OH} 43212$

\section{Bio}

Dr Dianne Morrison-Beedy, Professor at The Ohio State University, was inducted into the International Nursing Research Hall of Fame for HIPTeens: The Healt Improvement Project for Teens, recognised by the CDC and US Dept of Health \& Human Services as an exemplary evidence-based intervention for HIV/STI

Funding

titute of Nursing Research

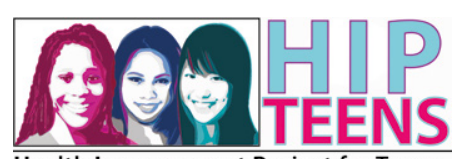

\section{References}

Morrison-Beedy, D, et al. 2013. Reducing sexual risk behaviour in adolescent gills. Resuls from a randomized htps:/www nchinlm nih gov/pmc/articles/PMC3580004/

Morrison-Beedy, D, et al. 2019. Adolescents in Scotland: Challenges and Opportunities for Sexual Risk Reduction. Journal of the Association of Nurses in AIDS Care 30(6):619-629

\section{Personal Response}

\section{Are there currently any plans to take HIPTeen
to other parts of the world?}

III HIPTeens is currently being disseminated to at-risk African, Caribbean, and Black Canadian teens and also and Burma. Work continues modifying HIPTeens for UK youth. As word of this brief, effective intervention spreads, we expect considerable movement into
global communities.

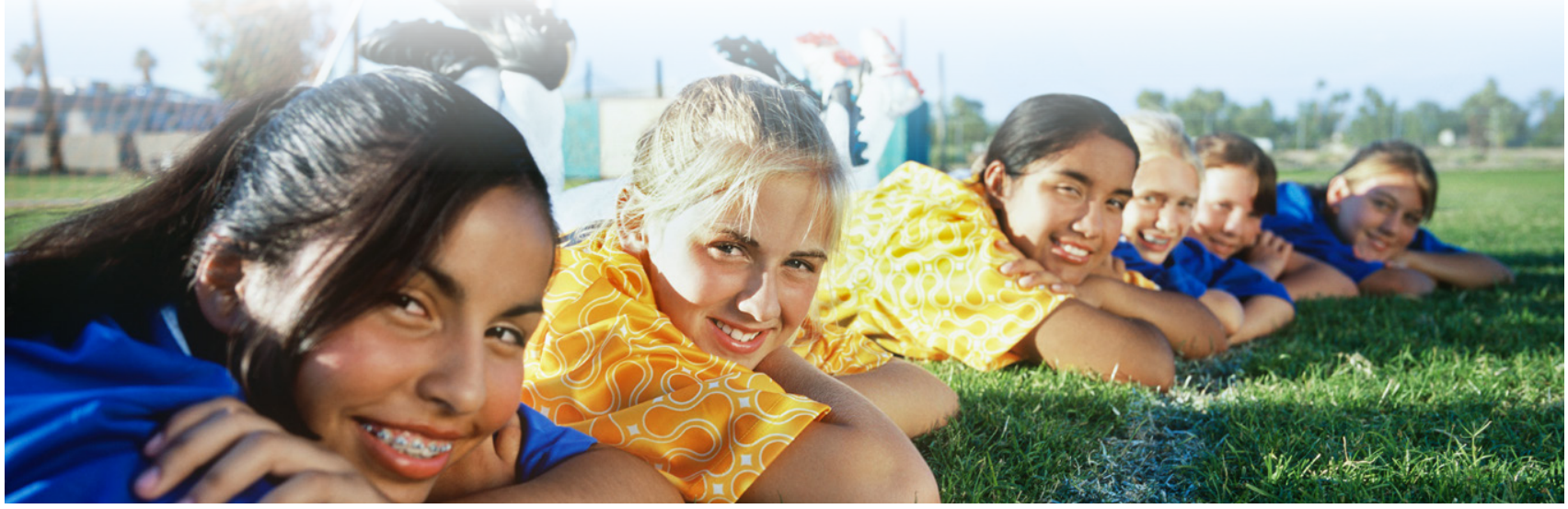

